cause. The secondary experimental end point was treatment goal achievement (TGA), defined as an integrated outcome.

Results: Among the 310 patients enrolled from $14 \mathrm{PAH}$ centers, the median follow-up was 24.0 months. The 1-, 3- and 5-year survival rates were $92.1 \%$, $84.8 \%$ and $72.9 \%$, respectively. The $1-$, 3 - and 5 -year TGA rates were $31.5 \%$, $53.6 \%$ and $62.7 \%$, respectively.Serositis, $6 \mathrm{MWD}>380 \mathrm{~m}$ and $\mathrm{Cl} \geq 2.5 \mathrm{~L} / \mathrm{min} \times \mathrm{m}^{2}$ were identified as independent prognostic factors of TGA. TGA within 5 years was identified as a factor associated with survival in patients with SLE-associated PAH (Figure 1).

Table 1. Baseline characteristics of patients with SLE-associated PAH

\begin{tabular}{lc}
\hline Characteristics & SLE-associated PAH $(\mathrm{n}=310)$ \\
\hline Age at recruitment, yr & $35.0 \pm 10.1$ \\
Female sex, \% & 99.4 \\
WHO Fc I-II, \% & 51.7 \\
GMWD, $\mathrm{m}$ & $408.6 \pm 98.0$ \\
$\mathrm{NT}-\mathrm{proBNP}, \mathrm{pg} / \mathrm{ml}$ & $1660.5 \pm 2275.1$ \\
SLEDAI & $6.1 \pm 5.5$ \\
$\mathrm{mPAP}, \mathrm{mmHg}$ & $46.5 \pm 12.1$ \\
$\mathrm{Cl}, \mathrm{L} / \mathrm{min} \times \mathrm{m}^{2}$ & $2.8 \pm 0.9$ \\
RAP, $\mathrm{mmHg}$ & $5.8 \pm 5.6$ \\
\hline
\end{tabular}

\begin{tabular}{|c|c|c|c|c|c|c|}
\hline \multirow[t]{2}{*}{ 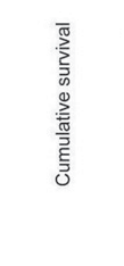 } & $20-$ & \multicolumn{5}{|c|}{$\begin{array}{l}\text {-Treatment goal achieved within } 5 \text { years } \\
\text { - - Treatment goal unachieved within } 5 \text { years }\end{array}$} \\
\hline & 0 & 12 & 24 & 36 & 48 & 60 \\
\hline No. at risk & \multicolumn{6}{|c|}{ Months from diagnosis } \\
\hline $\begin{array}{c}\text { Treatment goal } \\
\text { achieved }\end{array}$ & 111 & 92 & 68 & 47 & 27 & 15 \\
\hline $\begin{array}{l}\text { Treatment goal } \\
\text { unachieved }\end{array}$ & 152 & 101 & 69 & 41 & 29 & 17 \\
\hline
\end{tabular}

Conclusions: TGA was associated with the long-term survival, which supports and provides evidence to the treat-to-target strategy in SLE-associated PAH. Early diagnosis, intervention and heart function preservation are priorities for better long-term outcomes.

References:

[1] Badesch DB, Raskob GE, Elliott CG, Krichman AM, Farber HW, Frost AE, et al. Pulmonary arterial hypertension: baseline characteristics from the REVEAL Registry. Chest. 2010;137:376-87.

[2] Chung L, Liu J, Parsons L, Hassoun PM, McGoon M, Badesch DB, et al. Characterization of connective tissue disease-associated pulmonary arterial hypertension from REVEAL: identifying systemic sclerosis as a unique phenotype. Chest. 2010;138:1383-94.

[3] Condliffe R, Kiely DG, Peacock AJ, Corris PA, Gibbs JS, Vrapi F, et al. Connective tissue disease-associated pulmonary arterial hypertension in the modern treatment era. Am J Respir Crit Care Med. 2009;179:151-7.

Disclosure of Interest: None declared

DOI: 10.1136/annrheumdis-2017-eular.6718

\section{FRI0259 LUPUS LOW DISEASE ACTIVITY STATE (LLDAS) IN PATIENTS WITH LUPUS NEPHRITIS (LN)}

D.V. Monova, S. Monov, M. Ivanova, E. Peneva. Department of Internal Medicine, Medical Institute, Sofia, Bulgaria

Background: Lupus Low Disease Activity State (LLDAS) is a consensus-based definition of minimally acceptable disease activity in patients with Systemic lupus erythematosus (SLE).

Objectives: The aims of this study were to evaluate what proportion of patients with LN fulfils the definition of LLDAS and to evaluate the effect of LLDAS attainment on damage accrual over a period of 5 years.

Methods: This is a retrospective analysis of data prospectively collected in a longitudinal observational cohort of LN patients. The conceptual definition of LLDAS is a state, which if sustained, is associated with good long-term outcomes. The operational definition of LLDAS is fulfilled when all of the following criteria are met: (1) SLE Disease Activity Index (SLEDAI-2 K) $\leq 4$, with no activity in major organ systems (renal, central nervous system, cardiopulmonary, vasculitis, fever) and no haemolytic anaemia or gastrointestinal activity; (2) no new features of lupus disease activity compared to the previous assessment; (3) a Safety of Estrogens in Lupus Erythematosus National Assessment (SELENA)-SLEDAI physician global assessment (PGA) (scale 0-3) $\leq 1$; (4) a current prednisolone (or equivalent) dose $\leq 7,5 \mathrm{mg}$ daily; and (5) well-tolerated standard maintenance doses of immunosuppressive drugs and approved biologic agents, excluding investigational drugs. The definition of LLDAS was applied to each patient for each visit; organ damage was calculated with the SLICC/DI score (SDI) at study entry and at last observation.

Results: 294 patients were eligible for the study (89.79\% females, mean age at first visit $31,4 \pm 11,9$ years, mean disease duration at last visit $19,8 \pm 8,7$ years). At last observation 219 patients $(85,37 \%)$ were on treatment with glucocorticoids (GC) and/or immunomodulators. According with all the items of the definition, at last observation LLDAS was present in 146 patients (49,65\%); among these, 37 patients $(20,8 \%)$ maintained a stable LLDAS during the last 5 years of follow-up (LLDAS fulfilled for all visits). 68 patients (23,12\%) accrued organ damage during the follow-up; in the cohort as a whole the mean increase in SDI was $0,4 \pm 0.67$ resulting in a mean final SDI of $1,2 \pm 1,8$. Patients who maintained LLDAS were younger $(p<0,05)$, had a lower disease activity score at study entry $(p<0,001)$ and were more likely GC-free at last observation $(p<0,001)$. Patients who maintained LLDAS accrued less organ damage but this difference did not reach statistical significance.

Conclusions: High percentage of patients fulfils the proposed definitions for LLDAS at last visit but only a minority maintained this state for all the followup period. A minimally acceptable disease activity state is associated with a successful GC tapering and, probably, better long-term outcomes. LLDAS was shown to be associated with protection from damage accrual.

Disclosure of Interest: None declared

DOI: 10.1136/annrheumdis-2017-eular.1676

\section{FRI0260 ULTRASOUND STUDY OF PLEURAL PROFILE AND CHEST HIGH-RESOLUTION COMPUTED TOMOGRAPHY (HRCT): DIAGNOSTIC ROLE IN PRIMARY SJÖGREN'S SYNDROME-INDUCED LUNG INVOLVEMENT}

F. Ferro $^{1}$, A. Bulleri $^{2}$, A. Delle Sedie ${ }^{1}$, E. Elefante ${ }^{1}$, N. Luciano ${ }^{1}$, M. Mosca $^{1}$, C. Baldini ${ }^{1}$. ${ }^{1}$ Clinical and Experimental Medicine, Rheumatology Unit, University of Pisa; ${ }^{2}$ Radiology Unit, University of Pisa, Pisa, Italy

Background: Ultrasound pleural irregularity (PI-US) is a novel promising tool for non-invasive diagnosis of interstitial lung involvement (ILD) in connective tissue diseases (CTDs). Few data are available on its diagnostic usefulness in primary Sjögren's syndrome (pSS)-induced ILD.

Objectives: a) To assess the accuracy of PI-US to diagnose ILD in pSS when compared to chest tomography (HRCT) (i.e gold standard imaging technique); b) to explore PI-US diagnostic value in early preclinical phases of lung involvement. Methods: PI-US was performed by a single operator using a MyLab-25 (Esaote), $10 \mathrm{MHz}, 5 \mathrm{~cm}$ linear probe. PI was defined as the loss of the normal hyperechoic linear pleural contour (score 0-2: normal, minimal and major changes at each intercostal space). Abnormal findings at HRCT were quantified by an expert radiologist according to a semiquantitative score (0-2: absent, moderate, severe). Semi-quantitative scores assigned by PI-US and HRCT to 6 lung fields (2 for the anterior, 2 for postero-superior and 2 for postero-inferior chest surface) were compared. Total and partial scores (for each lung fields) were evaluated. For statistical analysis chi-square, Mann-Whitney test, R-Spearman, and ROC-curve analysis were used.

Results: Validation study phase (PI-US vs HRCT): To validate PI-US technique, we enrolled 32 pSS patients [M/F:5/27; median age (IQR): 67 yrs (51.5-71); median disease duration (IQR): 7 (4-11) yrs; anti-Ro/SSA (+) 78.1\%]. To be included patients should have performed a HRCT evaluation within 6 months. Thirteen patients $(41 \%)$ presented HRCT lesions suggestive for ILD. HRCT total scores and PI-US total scores were strongly correlated $(r=0.744, p=0.000)$ Similarly, PI-US and HRCT partial scores related to the postero-inferior fields showed a strong correlation one to each other $(r=0.780, p=0.000)$. ROC-curve analysis identified a total PI-US score of 28.5 (Youden index) as able to predict HRCT-diagnosis of ILD with a sensitivity (SE) of $84.6 \%$ and a specificity (SP) of $89.5 \%$. Analogously, a postero-inferior PI-US score of 12.5 demonstrated a SE of $100 \%$ and a SP of $89.5 \%$ for the ILD diagnosis. Prospective study phase exploring the usefulness of PI-US for ILD early pre-clinical diagnosis: We included 24 consecutive pSS patients without overt respiratory symptoms [M/F:1/23; median age (IQR): 55 yrs (47-67); median disease duration (IQR): 4 yrs (1-12); anti-Ro/SSA (+) 60.9\%]. Out of them, at the end of the diagnostic work -up, four new cases of HRCT-proven pSS-ILD were diagnosed. Their PI-US mean total score was significantly higher than that observed in non-ILD patients $(48 \pm 18$ vs $16 \pm 12, p=0.001)$ as well as their mean postero-inferior PI-US score ( $19 \pm 9$ vs $6 \pm 5, p=0.003)$. The total PI-US and postero-inferior PI-US cut-off retrieved in the first part of the study (i.e. 28.5 and 12.5) allowed us to identify those patients with an HRCT-proven pSS-ILD with a SE of $75 \%$ and $100 \%$, a SP of $95 \%$ and $89.5 \%$, a PPV of $75 \%$ and $57 \%$ and a NPV of of $95 \%$ and $100 \%$, respectively.

Conclusions: Even if preliminary, this study demonstrated a strong correlation between PI-US and HRCT in the detection of ILD-pSS also in asymptomatic patients, opening new perspectives for the early non-invasive screening of lung involvement in pSS.

Disclosure of Interest: None declared

DOI: 10.1136/annrheumdis-2017-eular.4368 